\title{
GABRIEL DEL BARCO: LA INFLUENCIA DE UN PINTOR ESPAÑOL EN LA AZULEJERÍA PORTUGUESA (1669-1701)
}

\author{
Maria do Rosário Salema de CARValho \\ Universidad de Lisboa
}

\begin{abstract}
Gabriel del Barco fue uno de los pintores de azulejos más significativos de cuantos trabajaron en Portugal a caballo de los siglos XVII y XVIII, momento en el que se estaba gestando una producción azulejera histórica para la caracterización de un arte que, con razón, ha sido identificado como uno de los más emblemáticos del patrimonio artístico portugués. Español de nacimiento, Gabriel del Barco llegó a Lisboa con casi veinte años. Se estableció en la ciudad, primero como pintor de techos y, más tarde, de azulejos. $\mathrm{Su}$ vida y obra, relativamente bien conocidas, han sido objeto de interés por parte de la historiografía portuguesa, que le ha dedicado algunos estudios, unos más enjundiosos que otros, pero, al cabo, todos ellos fragmentarios. El presente artículo pretende reunir las numerosas informaciones dispersas existentes sobre el pintor junto con datos desconocidos hasta nuestros días. Además de dar noticia de esa nueva documentación que ahora ve la luz, se procederá a hacer una reapreciación de la producción de Barco a través del análisis de los conjuntos cerámicos conservados con su firma y recurriendo al apoyo que las nuevas tecnologías ofrecen actualmente para la Historia del Arte.
\end{abstract}

Palabras clave: Azulejo; Azul y blanco; Pintura; Historiografía.

\section{GABRIEL DEL BARCO: THE INFLUENCE OF A SPANISH PAINTER ON PORTUGUESE TILE WORK (1669-1701)}

Gabriel del Barco was one of the most important tile painters working in Portugal during the transition from the $17^{\text {th }}$ to the $18^{\text {th }}$ centuries. This period is considered to be one of preparation preceding the great, emblematic era of Portuguese tile production during the $18^{\text {th }}$ century. Spanish born, Gabriel del Barco arrived in Lisbon when he was about twenty, and established himself there first as a painter of ceilings and later, as a tile painter. Articles on aspects of his life and work (relatively well-known), have been published, but they have provided only a fragmentary vision of this artist. Thus, this study unites numerous dispersed materials with the addition of heretofore-unknown documents concerning the painter. The author also offers a re-evaluation of the tiles painted by Barco by analyzing his signed tile panels, assisted by the use of new technologies.

Key words: Tiles; Blue \& white; Painting, Historiography.

Texto traducido del portugués por J. León Acosta.

Text translated from the Portuguese by J. León Acosta. 


\section{Introducción}

Gabriel del Barco fue un pintor de techos y de azulejos de origen español que llegó a Portugal en 1669, con casi 20 años de edad. Se estableció en Lisboa y permaneció en dicha ciudad al menos hasta 1701, fecha a la que remite la última noticia conocida sobre este artista que, durante los cerca de 30 años que vivió en la capital portuguesa, fue conocido como "Gabriel del Barco Hespanhol".

Cuando Barco llegó a Lisboa, la producción portuguesa de azulejos se caracterizaba por la vigencia de los modelos con patrones policromáticos, típicos del siglo XVII, aunque ya por entonces comenzaban a surgir paneles figurativos realizados por pintores con poca formación que, aunque inicialmente ingenuos, rápidamente evolucionaron hacia una pintura más erudita, caracterizada todavía por una intensa policromía. A partir de 1670 comenzaron a surgir los primeros ejemplos de paneles de azulejos en tonos de azul y blanco, o con mezcla de manganeso, dando así inicio a una moda que acabaría dominando el final de la centuria y adentrándose por toda la primera mitad del siglo XVIII. De esta forma se fraguaba uno de los periodos más importantes de la azulejería portuguesa.

Es precisamente en este contexto donde surge Gabriel del Barco, marcando de forma indeleble el periodo al que se ha dado en llamar "de transición" y sirviendo de eslabón entre la policromía característica del periodo anterior y el denominado Ciclo dos Mestres, esa fase de gran pujanza pictórica que coincide aproximadamente con el primer cuarto del siglo XVIII. En realidad, la historiografía ${ }^{1}$ portuguesa ha considerado al pintor español como el gran responsable de los cambios ocurridos a la sazón en la azulejería portuguesa, de cierta libertad gestual conseguida a través del equilibrio entre las manchas y el uso de líneas de contorno más suaves. José Meco, uno de los mayores especialistas portugueses en la materia, le atribuye un papel crucial ${ }^{2}$, llegando incluso a afirmar que fue él quien hizo posible el cambio en la azulejería ${ }^{3}$, al conseguir "una pintura específica a través de gradaciones expresivas de azul cobalto [...] junto con una escala

\footnotetext{
${ }^{1}$ Son muchas las referencias a Gabriel del Barco en el contexto de la historia de la azulejería portuguesa; no obstante, el número de estudios profundos es reducido. Habría que citar, casi exclusivamente, el primer esbozo de biografía escrito por Vergílio Correia en 1917, o las correcciones y nuevos datos divulgados por João Miguel dos Santos Simões en el volumen dedicado a la azulejería portuguesa del siglo XVIII. José Meco prestó especial atención a Gabriel del Barco -incluida su producción como pintor de techos y de azulejos policromáticos- desde una perspectiva de análisis muy llamativa, aunque poco consistente, razón que motivaría su abandono gradual de muchas de las atribuciones que en un principio había defendido. Véase también Viterbo, Francisco de Sousa, Notícia de alguns Pintores portugueses e de outros que, sendo estrangeiros, exerceram a sua arte em Portugal, Tomo I, Lisboa, Typographia da Academia Real das Sciencias, 1903, pp. 35-36; SequeIRA, Gustavo de Matos, Depois do Terramoto - Subsídios para a História dos Bairros Ocidentais de Lisboa, 2. ${ }^{a}$ ed., vol. IV, Lisboa, Academia das Ciências de Lisboa, 1867 [1934], p. 60; Correia, Vergílio, "Azulejadores e pintores de azulejos, de Lisboa", A Águia, n. 77 y 78, 1918, pp. 166-178; Santos, Reynaldo dos, O Azulejo em Portugal, Lisboa, Ed. Sul, 1957; Smith, Robert, "Três estudos bracarenses", Belas-Artes - Revista e Boletim da Academia Nacional de Belas Artes, 2. ${ }^{a}$ serie, n. ${ }^{\circ}$ 24-26, Lisboa, 1970, pp. 49-58; SimõES, João Miguel dos Santos, "Gabriel del Barco", Dicionário da Pintura Universal, Lisboa, Estudos Cor, 1974, p. 51; Idem, Azulejaria em Portugal no século XVIII, Lisboa, Fundação Calouste Gulbenkian, 1979; Meco, José, "O pintor de azulejos Gabriel del Barco", História e Sociedade, n. ${ }^{\circ}$ 6, diciembre de 1979, pp. 58-67 y n. 7 , mayo de 1981, pp. 41-50; Idem, Azulejos de Gabriel del Barco na região de Lisboa: período inicial, até cerca de 1691 - Pintura de tectos, separata del Boletim Cultural da Assembleia Distrital de Lisboa, III serie, n. ${ }^{\circ}$ 85, Lisboa, 1979; Idem, O Azulejo em Portugal, 2. " ed., Lisboa, Edições Alfa, 1993; Idem, "Barco, Gabriel del", Dicionário da Arte Barroca em Portugal, Lisboa, Editorial Presença, 1989, pp. 66-69.

2 Meco, José, “Azulejo”, Arte Portuguesa da Pré-História ao século XX, vol. 13, Lisboa, Fubu Editores, 2009, pp. 125-126.

3 Idem, “Azulejos de Gabriel del Barco...”, op. cit., 1979, p. 82.
} 
monumental de las composiciones, cada vez más grandiosas y complejas" ${ }^{\text {, }}$, animadas por un impresionante efecto escenográfico.

Limitar esta época de tan fecundas alteraciones a una sola personalidad y, paralelamente, hacerla responsable casi por completo de una evolución de tanto calado nos parece, en justicia, algo excesivo. La documentación que ha ido saliendo a la luz, junto con el inventario exhaustivo de los conjuntos cerámicos subsistentes, han ido demostrando la existencia de otros pintores y obradores de cerámica en activo a finales del siglo XVII, un hecho que prueba la vitalidad artística de la azulejería en la Lisboa de entonces ${ }^{5}$. Sin menoscabo del debido reconocimiento a Gabriel del Barco - que es unánime-, creemos que se impone estudiar su biografía y su obra en el contexto en que surgen, teniendo en cuenta las relaciones profesionales y familiares que lo ubican en la trama artística de la ciudad de Lisboa ${ }^{6}$.

\section{Antes de su llegada a Lisboa}

Hasta ahora tan sólo se sabía que Gabriel del Barco había nacido en Sigüenza, un municipio de la provincia de Guadalajara, a unos $150 \mathrm{~km}$ de Madrid, y que había llegado a Portugal en 1669 , cuando tenía ya casi veinte años. El reciente hallazgo de su partida de bautismo ${ }^{7}$ ha permitido fechar con mayor precisión el año de su nacimiento y, por ende, confirmar su filiación, que ya era conocida:

"En seis de diciembre de mil seiscientos cuarenta y ocho bauticé a Gabriel hijo de Manuel del Barco y de Polonia Palencia. Fueron sus compadres Gabriel de Palencia y Matea Pérez vecinos de esta ciudad, y lo firme / Br. Domingo de Laraz".

De su infancia y formación nada más se sabe, habiendo resultado imposible relacionar a ninguno de sus familiares con el medio artístico castellano de la época. La única excepción -y algo indirecta- reside en su relación con un paisano suyo, un tal Juan Calderón da Varca, imaginero, cuyo apellido presenta algunas similitudes con el de nuestro pintor. Algo que ya había notado Santos Simões ${ }^{8}$, quien subrayaba también la semejanza de nombres entre Barco y el célebre dramaturgo y poeta Pedro Calderón de la Barca (1600-1681).

${ }^{4}$ Idem, O Azulejo em Portugal, 2. ed., Lisboa, Edições Alfa, 1993, p. 216.

${ }_{5}$ Está en fase de redacción una tesis de doctorado sobre la materia, realizada por la autora de este artículo, quien ya ha publicado otro texto sobre el mismo asunto: CARvalho, Rosário Salema de, "Em torno de Gabriel del Barco-uma perspectiva sobre os pintores de azulejo no final do século XVII", Actas do colóquio Ver a Imagem-II Colóquio de Doutorandos em História da Arte, Ciências do Património e Teoria do Restauro, Lisboa, FLUL, 2010 (en imprenta).

${ }^{6}$ En un artículo de esta naturaleza no cabe hacer el análisis de este género de cuestiones, aunque estén permanentemente como telón de fondo. Hemos optado, pues, por reunir los datos conocidos sobre la figura de Gabriel del Barco y juntar algunos inéditos para, más tarde, analizar su obra firmada (14 conjuntos) y, así, poder ofrecer a la comunidad científica una base de trabajo rigurosa, susceptible de servir como punto de partida para una nueva apreciación de las obras atribuidas a este pintor, a quien le habría sido imposible -teniendo en cuenta los años en activo que se le conocen- haber pintado todas las obras que la historiografía le ha ido atribuyendo a lo largo de los años.

7 Archivo Histórico Diocesano de la Diócesis de Sigüenza - Guadalajara, Livro de bautismos, n. ${ }^{\circ}$, fl $82 \mathrm{v}$ [inédito].

8 Véase SimÕES, João Miguel dos Santos, op. cit., 1979, p. 20. Se debe a este investigador el descubrimiento de un importante documento que aporta los pocos detalles que se conocen de la vida de Barco durante este periodo, y que se deben a las cuestiones suscitadas por su casamiento, cuyo proceso forma parte de los Sumários Matrimoniais da Câmara Eclesiástica de Lisboa. Véase DGARQ/TT, Câmara Eclesiástica de Lisboa, Sumários Matrimoniais, mç. 696, n. ${ }^{\circ} 195,1669$. En el "proceso" constan las declaraciones de tres testigos que afirman haber conocido al pintor en Madrid, donde residía desde hacía tres años. Uno de ellos es el ya mencionado Juan Calderón da Varca, también natural de 
Sólo volveremos a recuperar la pista del pintor en torno a 1666/1667. Se sabe que a partir de entonces residió en Madrid. A qué se dedicaba y en qué círculos se movía son cuestiones aún por dilucidar. Lo que sabemos con seguridad es que ese medio le habría permitido acompañar en 1669 al primer embajador de Castilla, Charles Watteville ${ }^{9}$, en su viaje a Lisboa.

\section{La llegada a Lisboa}

Una vez en Lisboa, Gabriel del Barco se estableció en la parroquia lisboeta de Loreto. El 17 de julio de 1669 contrajo matrimonio con Agostinha das Neves, cuñada de uno de los pintores de caballete más prestigiosos de la ciudad, Marcos da Cruz, en cuya casa vivía la pareja o, al menos, la propia Agostinha, como se puede constatar por la partida de casamiento: "moradores en esta parroquia de Loreto, en la travesía Da Horta Seca, en las casas de Marcos da Cruz"10. Es precisamente en esta relación donde muchos investigadores han querido ver a Barco como dis-

Sigüenza. Los restantes eran Bartolomeu Fidalgo, oficial cochero, y Manuel de São Martinho, torcedor de seda, de 20 y 32 años, respectivamente, los cuales también formaban parte de la comitiva del embajador Charles de Watteville.

${ }^{9}$ El barón de Watteville, Bateville, o Vateville, pues son muchas las variantes gráficas de su apellido, fue un diplomático polémico nacido a principios del siglo XVII en Château-Vilaine (Nozeroy, Francia). Tras hacer carrera militar en Italia y haber participado en la pacificación de Nápoles, en 1660 fue enviado a la corte de Carlos II de Inglaterra como embajador del rey de España. Durante su estancia en Londres intentó evitar la boda entre el soberano inglés y Catalina de Braganza. Un año después protagonizaría un grave incidente diplomático con el embajador francés que acabaría por costarle el puesto de embajador. Solo en 1666, tras la muerte de Felipe IV, y una vez recuperada la confianza de la corte española, volverían a encargarle una misión, esta vez en Roma. En 1667 fue designado embajador del Sacro Imperio Romano Germánico, pero nunca llegaría a partir, pues la paz con Portugal lo llevaría a la capital portuguesa como primer embajador de Castilla.

De acuerdo con los datos conocidos, el embajador Charles de Watteville llegó a Portugal aún en el año 1668, aunque sólo sería recibido oficialmente tres meses más tarde, el 13 de febrero de 1669 (cf. Du Mont, Pierre, Le ceremonial diplomatique des cours de l'Europe, ou collection des actes, memoires et relations [...] recueilli en partie par Mr. Du Mont; mis en ordre et considerablement augmenté par Mr. Rousset, tomo II, Ámsterdam, chez les Janssons [etc.], 1739, p. 382; Faria, Ana Leal de, Arquitectos da Paz - A diplomacia portuguesa de 1640 a 1815, Lisboa, Tribuna da História, 2008). El barón, sin embargo, no permanecería mucho tiempo en Portugal, pues falleció en Lisboa en 1670 (cf. BlutEAu, Raphael, Oraçam funebre nas exequias do Excellentissimo senhor Barao de Bateville, Embaixador Extraordinario del Rey de Castela ao Principe nosso Senhor. Em presença dos tres estados da corte, prelados e Conventuaes das Religioens, na officina de Ioam da Costa, 1670). Los restos de Watteville se encuentran sepultados en la iglesia de Santa María della Passione, en Milán.

La figura de Watteville tampoco ha sido muy estudiada en España, donde tan sólo surge en algunas breves notas dispersas, la mayoría de ellas referentes a los acontecimientos ocurridos en Londres. Con todo, algunos datos nos permiten deducir que Watteville se relacionó con artistas de gran relevancia, entre los cuales se contaba el propio Velázquez, con el que estuvo en la isla de los Faisanes preparando el pabellón donde se firmaría el Tratado de Paz de los Pirineos, en 1659 (cf. Colomer, José Luis, "Paz política, rivalidad suntuaria - Francia y España en la isla de los Faisanes”, en Arte y diplomacia de la monarquía hispánica en el siglo XVII, 2003, pp. 61-88). También estuvo con el pintor Jacques Courtois el Borgoñón en las campañas militares del Milanesado, por un periodo de tres años (cf. SothEBY's, Old Master \& British Paintings Evening Sale, Londres, 9/12/2009). Ninguna de estas informaciones dejan traslucir los criterios seguidos por Watteville a la hora de elegir a los artistas que lo acompañarían en 1669, aunque sí nos permite entender que se moviese en un ambiente cultural que incluía a personalidades importantes, lo cual nos lleva a suponer que, aunque Gabriel del Barco fuese un joven al inicio de su carrera, no sólo tendría ya cierta formación, sino que ya habría dado pruebas de la misma en tierras españolas.

10 DGARQ/TT, Registos Paroquiais, Livro de Casamentos da Freguesia do Loreto (1668-1671), fl 27. Documento referido por Flor, Susana Varela de Almeida, 'Do seu tempo fazia parelha aos mais...', Marcos da Cruz e a Pintura Portuguesa do século XVII, Memoria de la Maestría en Arte, Patrimonio y Restauración, presentada en la Facultad de Letras de la Universidad de Lisboa en septiembre de 2002, vol. II, doc. 22. 
cípulo del pintor portugués ${ }^{11}$. No obstante, creemos que, aunque del Barco se haya beneficiado del contacto con artistas de prestigio establecidos en Lisboa, con los cuales incluso habría llegado a colaborar, su aprendizaje, que ciertamente habría tenido lugar en Madrid, estaba ya por entonces estabilizado.

En una primera fase, Gabriel del Barco se habría dedicado a pintar techos, una práctica muy extendida entre otros pintores de azulejos. Algunos investigadores ${ }^{12}$ han querido ver en esta formación de cariz más decorativo la causa de la evolución de los pintores de azulejos, su progresivo abandono de motivos recurrentes en favor de otros más libres, así como de grutescos. Se conocen dos documentos referentes a esta actividad: los recibos del año 1681, por los que se le abonaba el trabajo de pintura y dorado del techo del coro de la iglesia de san Luis Dos Franceses ${ }^{13}$ -residía entonces el pintor en la rúa Direita, que va de la iglesia del Loreto a Cruz de Pau-; y otro más tarde, en torno a 1689 , relativo a pinturas realizadas para la iglesia del convento de la Divina Providencia ${ }^{14}$. Ambos techos desaparecerían en 1755 con el terremoto, circunstancia que acaba por dificultar en gran medida la posibilidad de atribuir al pintor español ninguna obra de este género, ni siquiera considerando las hipotéticas semejanzas con los motivos pintados también en los paneles de azulejos, como había pretendido José Meco ${ }^{15}$.

Barco, en esa misma década de 1680, no sólo estaba perfectamente integrado en el medio artístico lisboeta, sino que además disfrutaba de un estatuto que le permitía ser admitido en la Hermandad de San Lucas, en la que estaba inscrito al menos desde $1683^{16}$. En realidad, en esta hermandad -con sede en el monasterio dominico de la Anunciada desde 1602, y que reunía a los pintores al óleo y al temple, además de pintores doradores y de estofados-, las discusiones giraban más en torno a problemas gremiales que a cuestiones de arte. Aunque no fuese, en puridad, una academia, por la hermandad pasaron los artistas más notables de la pintura al óleo de la época, un detalle que pone de manifiesto el prestigio que poseía cuando ingresó Gabriel del Barco ${ }^{17}$.

Otro acontecimiento ocurrido en $1681^{18}$, o al año siguiente ${ }^{19}$, prueba también la cómoda situación económica de la que gozaba Barco. Habiendo contraído una grave enfermedad, el pintor se encomendó a la Virgen de Atocha y, como agradecimiento por su intercesión, mandó copiar la talla existente en la capital española y colocarla en la iglesia del convento paulista de la sierra d'Ossa, sita en la calzada Do Combro. La ceremonia de entrega de la imagen tendría

11 Cf. Meco, José, “Azulejos de Gabriel del Barco...”, op. cit., 1979, p. 74; Idem, “O pintor de azulejos...”, op. cit., 1979, p. 59.

12 Entre ellos Meco, José, “Azulejos de Gabriel del Barco...”, op. cit., 1979, p. 81.

13 La documentación se encuentra inventariada en Alcochete, Nuno Daupiás, op. cit., 1958, pp. 59-60. Santos Simões consultó la documentación y refiere que los recibos datan de los días 3 y 15 de abril de 1681 y 4 de octubre de 1682. Cf. SimÕES, João Miguel dos Santos, op. cit., 1979, p. 20. La documentación de la Hermandad de San Luis dos Franceses se encuentra actualmente en el Centre des Archives Diplomatiques de La Courneuve, perteneciente al Ministerio de Asuntos Exteriores de Francia, razón por la cual tan sólo ha sido posible pedir las cotas referidas por Nuno Daupiás d'Alcochete, referentes a los pagos efectuados entre abril y julio de 1681, siendo que la lectura de algunas áreas resulta casi imposible.

${ }^{14}$ Cf. Meco, José, “Azulejos de Gabriel del Barco...”, op. cit., 1979, p. 47; Idem, “O pintor de azulejos...”, op. cit., 1979, p. 59.

15 Cf. Idem, “O pintor de azulejos...”, op. cit., 1979 y 1980; Idem, op. cit., 1979.

16 Cf. TeiXeira, F. A. Garcez, op. cit., 1931, p. 125.

17 Cf. SERrão, Vítor, A Pintura Protobarroca em Portugal 1612-1657 - o Triunfo do Naturalismo e do tenebrismo, Lisboa, Edições Colibri, 2000, pp. 210 y ss.

${ }_{18}$ Cf. Costa, António Carvalho da, Corografia Portuguesa..., tomo III, Lisboa, Officina Real Deslandesiana, 1712 , p. 491, referido por SiMÕES, op. cit., 1979, p. 20 y MECO, José, “O pintor de azulejos [...], op. cit., 1981, pp. 48-49.

19 Cf. Santa Maria, Agostinho de, Santuário Mariano, Livro Segundo, 2.a ed., Lisboa, Miscelánea, 1933, pp. 345-347.

Arch. esp. arte, LXXXIV, 335, JULIO-SEPTIEMBRE 2011, 227-244, ISSN: 0004-0428 
lugar en 1683, acompañada de una muy concurrida y suntuosa procesión ${ }^{20}$. Su devoción daría lugar a una cofradía encargada de cuidar la capilla, una de las "más graves y aseadas que tiene esta corte" 21 .

En 1689, Gabriel del Barco firma como testigo instrumental22 y es designado como pintor en el testamento de Francisco Lopes Ferrão - casado con Isabel Soares y moradores en la rúa Do Caldeira, en el barrio de Santa Catarina-. La primera pintura sobre azulejo firmada por Barco de la que se tiene noticia aparece fechada, precisamente, dos años más tarde, en 1691 (v. infra lista de los conjuntos cerámicos).

Como venimos constatando, las informaciones relativas a Gabriel del Barco son más bien escasas, en particular todo lo concerniente a su actividad artística, que incluye la pintura de techos (documentada), la probable pintura de caballete (nunca demostrada) y la pintura de azulejos, concentrada en la última década del siglo XVII y de la cual se conocen quince conjuntos cuya autoría nadie pone en tela de juicio.

Una vez más, falta por dilucidar cuál fue su formación en el área de la azulejería, actividad cuyo arranque sitúa Santos Simões hacia 1687, apoyándose en el hecho de que ese año se hubiese decretado el embargo a las importaciones de azulejos holandeses, cuya consecuencia inmediata fue la expansión de la manufactura cerámica portuguesa ${ }^{23}$. Por su parte, José $\mathrm{Meco}^{24}$ plantea la posibilidad de que Barco hubiese aprendido a pintar azulejos en las alfarerías populares, participando en obras policromáticas, pero siempre como resultado de la colaboración entre diferentes obradores, sin voluntad expresa de autoría. En una fase que dicho investigador considera inicial e inmediatamente anterior a las primeras obras firmadas, atribuye a Barco obras ornamentales pintadas sólo con azul y blanco ${ }^{25}$, uniendo así su pintura de azulejos con la de techos.

Lo cierto es que en 1691, Gabriel del Barco contaba ya con cuarenta y tres años, una edad avanzada para iniciarse en cualquier modalidad artística si no se ha tenido ninguna experiencia previa. Así pues, consideramos como muy probable que se hubiese ejercitado en la pintura policromática, pero ello no implica que se le pueda atribuir ésta o aquella obra. Lo mismo ocurre con las pinturas de motivos ornamentales en azul y blanco, o las de patrón, cuya producción en serie dificulta los intentos de individualización ${ }^{26}$. Por otra parte, no hay que olvidar el contacto del pintor con obras procedentes del norte de Europa, como se puede apreciar en la iglesia de Madre de Deus, en la que se le ha atribuido la autoría de un paño del zócalo, o en las casas de Domingos Dantas da Cunha, en el Torel, una zona de la céntrica parroquia de São José (Lisboa). En ese sentido, no cabe duda de que, una vez instalado en la ciudad ${ }^{27}$, habría tenido la oportunidad de contemplar los paneles que cubrían las salas del palacio de los marqueses De Fronteira.

La primera obra de Gabriel del Barco, firmada y fechada en 1691, es la capilla de san Juan Bautista, en la Quinta de Nossa Senhora da Conceição, en Barcarena (fig. 1). De ese mismo año existe un documento de origen desconocido en el que Barco aparece con un apellido diferente, Gabriel del Barco y Minusca, un elemento que no se volverá a repetir ${ }^{28}$ en adelante.

${ }^{20}$ Idem, ibidem. Nótese que la iglesia de los paulistas se concluyó en 1680 y que fue entonces cuando se iniciaron las campañas decorativas, en las cuales parece haber estado implicado Gabriel del Barco.

${ }^{21}$ Cf. Costa, António Carvalho da, op. cit., p. 491.

22 DGARQ/TT, Registo Geral de Testamentos, Liv. 67, fls. 37-38 [inédito].

23 Cf. SimõES, João Miguel dos Santos, op. cit., 1979, p. 21.

${ }^{24}$ Cf. Meco, José, “Azulejos de Gabriel del Barco...”, op. cit., 1979, p. 79; Idem, “O pintor de azulejos...” op. cit., 1979, p. 59; Idem, "Azulejos de Lisboa", en Azulejos de Lisboa: exposição, promovida por el Ayuntamiento de Lisboa; responsable de la organización, Museu da Cidade; programa, selección y catálogo, José Meco. Lisboa, C.M., 1984, p. 53.

${ }^{25}$ Cf. Idem, ibidem, p. 59.

${ }^{26}$ Para una concepción diferente, véase Idem, "O pintor de azulejos...” op. cit., 1979, p. 43.

${ }^{27}$ Cf. Idem, "Azulejos de Gabriel del Barco...", op. cit., 1979, p. 78.

28 Cf. Correia, Vergílio, op. cit., 1918, p. 169. 


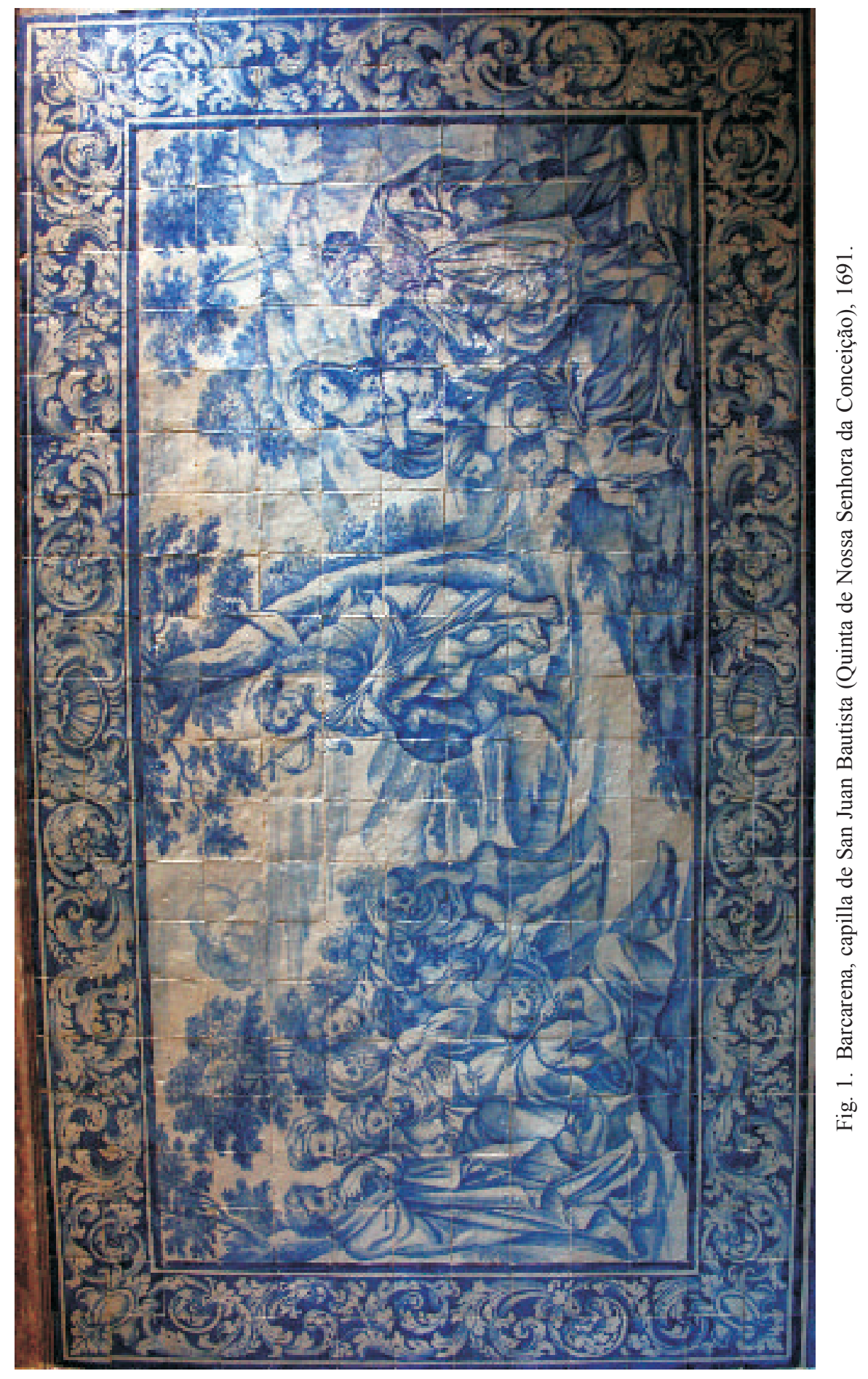

Arch. esp. arte, LXXXIV, 335, JULIO-SEPTIEMBRE 2011, 227-244, ISSN: 0004-0428 
En 1692 encontramos a Barco en la zona de Poiais de Jesus, más precisamente en la rúa Poiais de São Bento, donde fue vecino de Francisco Ferreira de Araújo ${ }^{29}$, pintor de techos que también pertenecía a esta gran familia de artistas. En 1695 muda de nuevo de domicilio y lo localizamos en la rúa Das Parreiras. Con él vivían Brás da Costa, casado con Maria dos Santos, hijo de Manuel de Almeida y de Antónia da Costa, propietarios de un horno instalado en la segunda travesía Dos Poiais ${ }^{30}$. Ese mismo año realizaría los paneles del palacio de Domingos Dantas da Cunha, en Lisboa ${ }^{31}$ y otro conjunto del que ha llegado hasta nuestros días parte de un panel en la Casa de Santar, en Nelas ${ }^{32}$, cuya firma se encuentra en un único azulejo, conservado en el Museo Nacional del Azulejo (MNAz) con el número de inventario 402. En 1696 llevó a cabo el revestimiento de la capilla mayor de la iglesia de san Bartolomé, en Charneca do Lumiar (Lisboa). Ese mismo año se mudaba de la parroquia de Santa Catarina a la de Santos-o-Velho -concretamente, a la rúa Do Olival- y entraba en la Hermandad del Santísimo Sacramento ${ }^{33}$.

$\mathrm{Al}$ año siguiente pintó tres grandes conjuntos. De uno de ellos se desconoce su paradero o si han quedado vestigios. De los dos restantes tan sólo se conocen algunos paneles dispersos. Para el palacio de los condes Da Ponte realizó temas alegóricos ${ }^{34}$; para el edificio del largo del Terreiro do Trigo ${ }^{35}$ pintó motivos venatorios y para la celda de los priores generales del monasterio de santa María de Belém, varios paneles de temática desconocida, pues han desaparecido ${ }^{36}$. El documento referente a esta última obra, escrito por el propio Gabriel del Barco, está fechado a 19 de marzo de 1697. El pintor, poniéndose a sí mismo y a sus bienes como aval, se compromete en él a tener los azulejos acabados a mediados del mes de abril. Sigue a esta promesa una suerte de esquema contable en el que se puede leer "dibujar todo ------ 14\$200", es decir, una parcela relativa a la concepción de la obra.

De 1698 datan sus trabajos para la iglesia de Nuestra Señora Dos Prazeres de Beja y la casa de la calzada Dos Cavaleiros -o De Santo André-, en Lisboa ${ }^{37}$. En 1699 pintó los paños monumentales de la iglesia de Santiago de Évora. Entre ese año y el siguiente realizaría también el conjunto monumental de la iglesia del antiguo convento de los Loios (Arraiolos), así como los paneles de una casa ubicada en la zona lisboeta de São Bento ${ }^{38}$, los de una capilla particular de la ciudad de Portalegre (fig. 2) y el conjunto de la sala de la Hermandad del Santísimo Sacramento de la iglesia de São Mamede en Évora ${ }^{39}$. Las matrículas de confesados indican que en 1699 se mantenía en el mismo domicilio, donde también vivía alguien llamado Luis, al cual Vergílio Correia consideró su aprendiz ${ }^{40}$. Sin embargo, en 1700 ya había mudado nuevamente de casa y aparece como morador en la rúa Da Oliveira, en la parte de levante ${ }^{41}$.

${ }^{29}$ Cf. Idem, ibidem, p. 169.

30 Cf. Idem, ibidem, p. 169.

31 Cf. Simões, João Miguel dos Santos, Carreaux Céramiques Hollandais au Portugal et en Espagne, La Haye, Martinus Nÿhoff, 1959, p. 33; MECO, “O pintor de azulejos...” [op. cit.], 1979, p. 63.

${ }^{32}$ Cf. SimõES, João Miguel dos Santos, Azulejaria em Portugal no século XVIII-ediçãó revista e actualizada, Lisboa, Calouste Gulbenkian, 2010, p. 173, nota 465 de Patricia Roque de Almeida.

${ }^{33}$ Cf. Idem, ibidem, p. 169 y 175.

34 Tan sólo se conoce el panel que representa una figura alegórica perteneciente a la colección Herederos del Dr. José d'Alpoim, en depósito en el MNAz.

35 Panel perteneciente al fondo del Museo de San Roque, Lisboa.

${ }^{36}$ DGARQ/TT, Monasterio de Santa María de Belém, mç. 4, doc. 59, n. 21. Documento divulgado por Meco, José, “A azulejaria e a cerâmica escultórica nos Jerónimos”, en Jerónimos 4 séculos de pintura, vol. I, Lisboa, MC/IPPAR, p. 119.

37 De este conjunto quedan algunos paneles dispersos. El depositado en el MNAz tiene el n. ${ }^{\circ}$ de inventario 900; el del hotel Sheraton fue destruido en 1996.

${ }^{38}$ De esta obra sólo han llegado hasta nosotros los restos de las figuras de Céfalo y Procris, MNAz, inv. 373 y 373 a.

39 Cf. SimÕES, João Miguel dos Santos, op. cit., 2010, p. 529, nota 1365 de Maria Teresa Verão.

${ }^{40}$ Cf. Correia, Vergílio, op. cit., 1918, p. 169 y Arquivo da Igreja de Santos-o-Velho, A3 P1 M. 01 SV1, fl 58 v.

${ }^{41}$ Cf. Correia, Vergílio, op. cit., 1918, p. 169. 


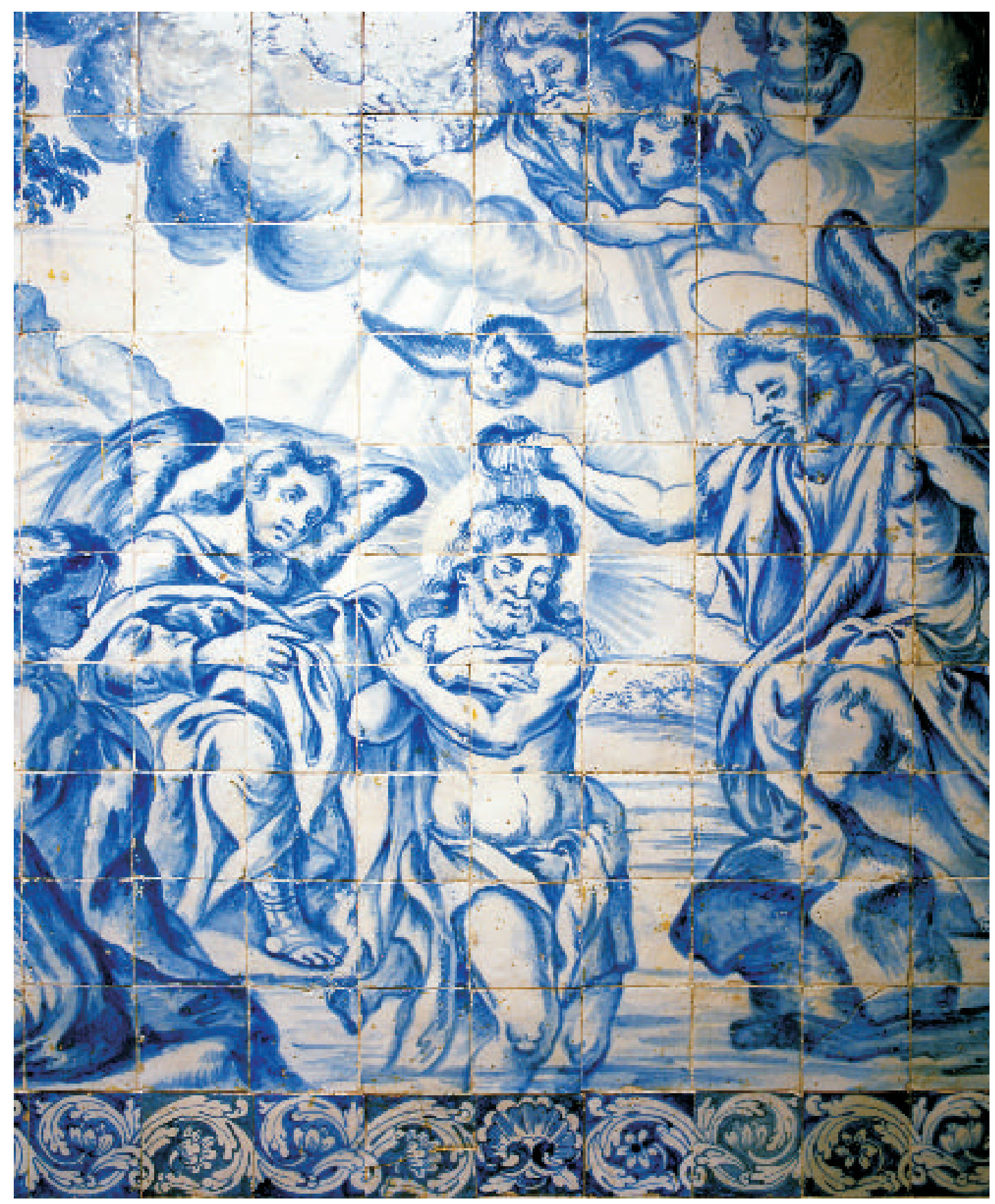

Fig. 2. Portalegre, capilla particular, 1700 (fotografía cedida por Teresa Saporiti). 




Fig. 3. Lisboa, palacio de la Independencia, 1696.

Tanto los trabajos realizados para el convento de los Loios (fig. 4) como los de la casa de la rúa São Bento o de Portalegre son las últimas obras conocidas de Gabriel del Barco. El 18 de enero de 1701 fallecía Agostinha das Neves, su esposa, cuando vivían ambos junto al monasterio Das Inglesinhas. A pesar de que en su certificado de óbito se indicase que había dejado testamento, y que en él se nombraba heredero a su marido, ese documento ${ }^{42}$ aún no se ha podido localizar. Agostinha das Neves fue enterrada en "la sepultura de Nuestra Señora Da Tocha [de Atocha], sita en la iglesia de los religiosos paulistas de esta ciudad"43, o sea, el altar mandado construir unos años antes por el propio Barco. En la matrícula de confesados de ese año, Gabriel del Barco aparece como viudo y residente en la travesía Das Inglesas, donde todavía vivía cuando el 18 de abril de ese año se volvió a casar, esta vez con Maria Teresa Baptista, hija legítima de Francisco Baptista y Maria Simões, natural de la parroquia de San Lourenço, en el término municipal de la villa de Azeitão ${ }^{44}$.

A partir de esta fecha se le pierde el rastro a nuestro autor. Hay quien defiende que regresó a España ${ }^{45}$; otros, que continuó pintando hasta por lo menos 1703 , año en que habría dejado incompleta la obra de la iglesia mayor de Sardoal, que le ha sido atribuida parcialmente ${ }^{46}$. Santos

${ }^{42}$ Cf. Idem, op. cit., 1918, p. 169. DGARQ/TT, registro parroquial de Santos-o-Velho (Óbitos), fl 78, MF1166.

43 DGARQ/TT, registro parroquial de Santos-o-Velho (Óbitos), fl 78, MF1166.

44 Cf. CorreIA, Vergílio, op. cit., 1918, p. 169. DGARQ/TT, registro parroquial de Santos-o-Velho (Matrimonios), fl 66 e 66 v, MF1160.

${ }^{45}$ Cf. Correia, Vergílio, op. cit., 1918, p. 169.

${ }^{46}$ Cf. Meco, José, “Azulejos de Gabriel del Barco...”, op. cit., 1979, p. 75; Idem, “O pintor de azulejos...” op. cit., 1979, p. 60. 




Fig. 4. Arraiolos, iglesia del antiguo convento de los Loios, 1700

(fotografía de Francisco Queiroz/Instituto de Promoción Cerámica).

Simões se inclinaba por considerar que Barco se mantuvo en activo hasta 1708 , pero no presenta ninguna prueba que justifique esa fecha ${ }^{47}$. Lo cierto, a pesar de todo, es que no existen datos que puedan corroborar ninguna de esas hipótesis. Tan sólo la referencia de fray Agostinho de Santa María parece arrojar alguna luz sobre esta cuestión. En 1707 se lamentaba dicho religioso de la falta de entusiasmo de los feligreses en la devoción a la Virgen de Atocha desde que faltara Gabriel del $\mathrm{Barco}^{48}$, una afirmación de la que se infiere que el pintor o no vivía ya en Lisboa o había fallecido.

Observando los datos conocidos sobre la vida de Gabriel del Barco se concluye que, en un periodo de aproximadamente treinta años, mudó de casa al menos siete veces. Como pintor de azulejos, su producción de composiciones exclusivamente en azul y blanco se localiza en el periodo final de su vida, cumplidos los cuarenta y tres años, y se prolonga a lo largo de una década escasa. Sin embargo, durante ese tiempo, y concretamente a partir de 1695, su producción azulejera conocida arroja una media de dos a tres obras por año, algo que, teniendo en cuenta la monumentalidad de muchas de ellas - particularmente las últimas-, pone de manifiesto el intenso ritmo de producción de Gabriel del Barco, o del obrador que dirigía.

47 Cf. SimÕES, João Miguel dos Santos, 1979, p. 20.

48 Cf. Santa Maria, Agostinho de, op. cit., pp. 345-347. Fue José Meco quien alertó sobre este pormenor, cf. Meco, José, “O pintor de azulejos...” op. cit., 1980, p. 49. 
Antes de pasar al análisis de las obras señaladas, vale la pena introducir una nota a propósito de las firmas utilizadas por el autor, cuyas abreviaturas surgen aquí y allá con diferencias significativas. En los azulejos de Barcarena, el nombre de Gabriel del Barco va precedido por una $D$., seguramente como abreviatura de Don. Su nombre escrito por extenso aparece en este panel, en el n. ${ }^{\circ} 402$ del MNAz -donde no se ve el primer nombre, aunque el apellido Barco está completo-, en uno de los paneles del palacio de la Independencia, en la iglesia de Santiago de Évora, en el convento de los Loios de Arraiolos (fig. 5) -en las dos firmas, pues, aunque la del azulejo de la entrada esté truncada, todavía se puede leer briel del / arco F 1699- y en el panel n. ${ }^{\circ} 373$ del MNAz.

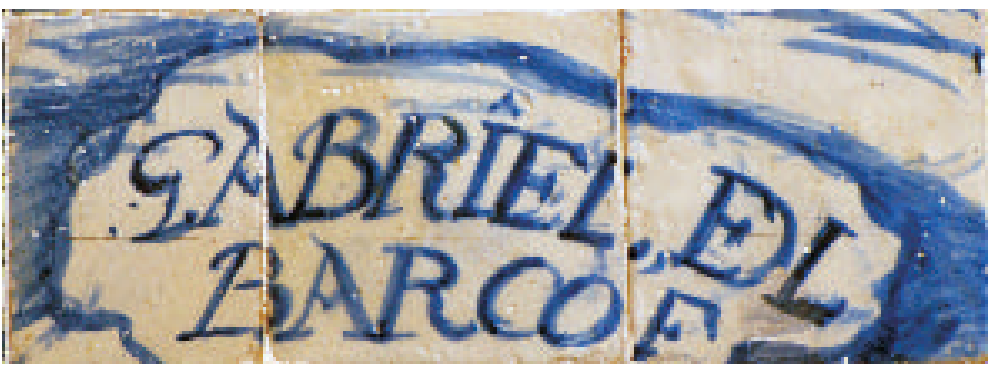

Fig. 5. Arraiolos, iglesia del antiguo convento de los Loios, 1699/1700, pormenor de la firma de Gabriel del Barco. Salvo el panel oriundo de una casa situada en el largo del Terreiro do Trigo (Lisboa) y otro del palacio de la Independencia -ilegible-, las restantes firmas terminan todas con la letra $F$, abreviatura de $f e z$ ofecit que corrobora la autoría. Así y todo, en la iglesia de São Mamede (Évora) la palabra $f e z$ es perfectamente legible.

\section{Características de la pintura de Gabriel del Barco}

Al comparar las consideraciones sobre la pintura de Gabriel del Barco vertidas por los diferentes autores que se han dedicado a estudiar su obra, constatamos que todos ellos son unánimes a la hora de clasificarlo como mal dibujante a la par que excelente pintor, destacando el sentido y el efecto decorativos que emanan de sus trabajos ${ }^{49}$. Reinaldo dos Santos, al caracterizarlo, emplea expresiones como "sin elegancia, sin ritmo, de proporciones achatadas, de dibujo esquemático y tosco" 50 . Así y todo, destaca su uso del azul intenso, que imprime fuerza y un gran efecto decorativo. Santos Simões hace referencia a su "dibujo libre, casi sintético, pero con una perspectiva poco correcta, con mucho contorno y zonas del cuerpo dibujadas de forma grosera" ${ }^{\text {. }}$. José Meco es quien más defiende las características específicas de Gabriel del Barco, cuya pintura considera espontánea y densa, aunque con un dibujo pobre y con errores de perspectiva ${ }^{52}$. Para este especialista, su pintura tiende a liberarse del trazo y a alcanzar una expresión muy singular en la pincelada suelta y en la mancha directa, detalles que reflejan la preocupación de

49 Además de los autores mencionados en las notas siguientes, véase también SEQUEIRA, Gustavo de Matos, op. cit., 1967 [1934], p. 60; Sмiтн, Robert, The Art of Portugal (cerámica), Londres, Weidenfeld and Nicolson, 1968, p. 233; Idem, op. cit., 1970, p. 49; MANGUCCI, Celso, "A manufactura e a pintura de azulejos em Portugal da produção das primeiras faianças à grande indústria oitocentista", O Revestimento Cerâmico na Arquitectura em Portugal, Lisboa, Estar/ Recer, 1998, pp. 19-64; SERRão, Vítor, História da Arte em Portugal - o Barroco, Barcarena, Editorial Presença, 2003.

50 SANTOS, Reinaldo dos, op. cit. 1957, p. 112.

51 SIMÕES, João Miguel dos Santos, op. cit., 1979, p. 22.

52 Cf. Meco, José, “Azulejos de Gabriel del Barco...", op. cit., 1979, p. 82; Idem, "O pintor de azulejos...” op. cit., 1979, pp. 58-67 y 1980, pp. 41-50; Idem, "Azulejos de Lisboa” op. cit., 1984, pp. 15-82; Idem, "Barco, Gabriel del" op. cit., 1989, pp. 66-69; Idem, "Le 'cycle des maitres' baroques", en Azulejos, catálogo de la exposición presentada en Europália 91, Bruselas: Europalia 91, 1991, pp. 41-48; Idem, “Azulejo” op. cit., 2009, pp. 111-142. 
Barco por la expresividad, así como cierto desinterés por la corrección del dibujo, que relega a un segundo plano.

De las lecturas anteriores se desprende, a nuestro entender, una visión excesivamente generalista que, salvo contadas excepciones, apenas ha recibido un análisis en profundidad y que, por lo demás, no ha tenido como punto de partida un tratamiento sistemático de la obra cuya autoría se puede atribuir a Gabriel del Barco sin discusión. Definir el corpus artístico de un pintor determinado implica identificar su técnica, las peculiaridades que se mantienen y las alteraciones que van siendo introducidas, o los modelos, todo ello desde una perspectiva cronológica que permita, además, percibir los derroteros por los que va evolucionando a lo largo de su carrera. Eso es lo que hemos pretendido hacer con la obra de Barco, observando cuidadosamente cada uno de los conjuntos de azulejos que listamos a continuación para facilitar la lectura ${ }^{53}$. Además de los catorce conjuntos que han llegado hasta nosotros, incluimos también el del monasterio de santa María de Belém, que acabaría por desaparecer.

1691 - Barcarena, Quinta de Nossa Senhora da Conceição, capilla de san Juan Bautista 1695 - Lisboa, Palacio de Domingos Dantas da Cunha, jardín en la cuesta del Torel 1695 - Lisboa, panel de azulejo (fragmento), MNAz, inv. ${ }^{\circ} 402$

1696 - Lisboa, Palacio de la Independencia, dos paños aplicados en la terraza superior 1696 - Lisboa, capilla mayor de la Iglesia de San Bartolomé, Charneca do Lumiar

1697 - Lisboa, Palacio de los Condes da Ponte [un panel perteneciente actualmente a la Casa da Anha (Viana do Castelo), en depósito en el MNAz]

1697 - Lisboa, Museo de San Roque, procedente de una casa del largo del Terreiro do Trigo

1697 - Lisboa, Monasterio de Santa María de Belém, dormitorios, celda de los priores generales del monasterio [desaparecida]

1698 - Lisboa, casa de la calzada Dos Cavaleiros | De Santo André [adquiridos por José Manuel Leitão - un panel en el MNAz (inv. 900) que representa a Iris y Junot; dos en el hotel Sheraton [desaparecidos]; col. Jorge de Brito, Cascáis; col. Óscar Husum, Malveira da Serra; col. José Manuel Leitão]

1698 - Beja, Iglesia de Nossa Senhora dos Prazeres

1699[?] - Évora, sala de la Hermandad del Santísimo Sacramento de la iglesia de São Mamede

1699 - Évora, Iglesia de Santiago

1699/1700 - Arraiolos, iglesia del antiguo Convento de los Lóios

1700 - Lisboa, panel de azulejos con las figuras de Céfalo y Procris, MNAz (inv. 373 y 373A)

1700 - Portalegre, capilla particular

En todos estos paneles se percibe cierto parentesco y una forma de pintar aparentemente consistente que se repite sin alteraciones significativas, expresada con una técnica de ejecución

53 Dentro del ámbito de la tesis de doctorado, se ha efectuado un inventario exhaustivo de las obras firmadas y atribuidas a Gabriel del Barco, un proceso que se inició con sendas visitas a cada uno de los lugares identificados, durante las cuales se fue construyendo un archivo fotográfico de los paneles y de los detalles considerados más relevantes. Para ese efecto se ha utilizado una máquina fotográfica digital de alta precisión (Canon EOS 450D, de 12 megapixels); para su reproducción se ha recurrido a pantallas de alta resolución. Sólo así se ha conseguido detectar diferencias técnicas y de detalle. Sin este valioso apoyo técnico -que no estuvo al alcance de los investigadores que nos precedieron-, habría sido imposible realizar esta tarea. El inventario de los conjuntos de azulejos se llevó a cabo en una base de datos construida en FileMaker por la empresa Peopleware, una aplicación que nos ha permitido organizar todos los registros, facilitando así las comparaciones y asociaciones. 
muy ágil y rápida. Sin embargo, tras un análisis más atento se perciben múltiples variaciones, incluso entre paños pertenecientes al mismo conjunto.

En la capilla de Barcarena (fig. 1), la primera obra de Barco, se observa un uso no muy acentuado del contorno; un dibujo de los volúmenes y las sombras -a veces un poco manchados- resuelto mediante pinceladas paralelas con aguada; un tratamiento de los tejidos en el que predomina la mancha; rostros masculinos escuálidos y toscos, por contraste con los femeninos, más trabajados; y unas proporciones de las figuras humanas poco conseguidas, aunque con una definición clara de la musculatura.

Las obras de 1695 en los jardines del palacio de Domingos Dantas da Cunha y el panel de la Casa de Santar no permiten evaluar correctamente la técnica de Barco, pues este último, muy fragmentario, está mal aplicado, y el primero se encuentra ya muy deteriorado.

$\mathrm{Al}$ año siguiente, en 1696, en dos obras firmadas y fechadas -iglesia de san Bartolomé (Charneca do Lumiar) y palacio de la Independencia (fig. 3)-, se encuentran las grandes tendencias que marcan los trabajos de Gabriel del Barco, muy dispares entre sí, curiosamente. En la capilla mayor del templo de Lumiar predomina el recurso a la pincelada paralela, más fina, con un sentido de ordenación atenuado. En puridad, lo que sobresale es la rapidez de ejecución y la consiguiente falta de contención, que a menudo casi transforma trazos separados en manchas. El dinamismo es tal que, en los fondos de paisajes rocosos, montañas o troncos de árboles, el resultado final recuerda permanentemente los efectos de la pintura impresionista. Los perfiles de las figuras son gruesos, confundiéndose muchas veces con los fondos, pero la caracterización de los rostros está cuidada, es expresiva, pese a su pincelada esquemática. Si comparamos este conjunto con el del palacio de la Independencia, la diferencia es extraordinaria, pues los reducidos personajes de cazadores que aparecen en este último fueron pintados de forma sintética, con un trazo de contornos finos, a veces repetido, casi como si se tratase de un boceto al carbón. Los volúmenes están sugeridos con pinceladas paralelas, anchas y distantes entre sí, dejando bien visibles las aguadas que las complementan. Los rostros masculinos son idénticos a muchos de los que aparecen en obras firmadas por Barco, aunque, por contraste, aquí la línea destaca sobre la mancha, que suele ser más habitual. De cierta forma, estos dos paneles que han llegado hasta nuestros días evocan una pintura cercana a la caricatura, lejos del "impresionismo" que habíamos señalado en piezas anteriores.

Las obras siguientes confluirán con la tendencia que se adivinaba en la iglesia de san Bartolomé (Charneca do Lumiar), aunque en varios casos es posible entrever elementos presentes ya en el palacio de la Independencia, pues las técnicas se mezclan y conjugan. Ya en 1697 volvemos a depararnos con las mismas incongruencias. En efecto, el fragmento de panel oriundo del edificio situado en el largo del Terreiro do Trigo (Lisboa) es idéntico a los dos del palacio de la Independencia. Por otro lado, la figura alegórica que formaba parte del revestimiento del palacio de los condes Da Ponte (fig. 6), en la que Barco recurre a una pintura dominada por el uso de manchas, con contornos prácticamente diluidos sobre el fondo y una pincelada suelta, se destaca como el mejor ejemplo su producción.

Los trabajos siguientes presentan, como ya se ha dicho, esa dualidad entre pinceladas paralelas de gran dinamismo y apuntes más contenidos. Aún habría que reseñar que, en la iglesia de Nuestra Señora Dos Prazeres de Beja, una de las obras de Barco que suscita más interrogantes, las diferencias entre los paneles son notorias, combinando figuras con un alto grado de pormenor a la par que otras en las que predomina el trazo esquemático, especialmente en los rostros femeninos. La falta de coherencia sugiere, incluso, la probable participación de uno o más colaboradores. No obstante, y dado que muchos de esos rostros están también presentes en paneles de otros conjuntos, no excluimos la posibilidad de que respondan a intenciones o a formas de pintar diferentes, pues nada impide que el mismo pintor se decidiese por explorar más de un lenguaje. Así y todo, también cabe la posibilidad de que Gabriel del Barco haya colaborado en más de una ocasión con los mismos artistas, lo cual sería una explicación razonable para estas repeticiones. 




Fig. 6. Lisboa, panel procedente del palacio de los condes Da Ponte, 1697 (C) Paulo Cintra | Laura Castro Caldas).

En la sala de la Hermandad del Santísimo Sacramento de la iglesia de São Mamede (Évora) se observa, una vez más, cierta incoherencia, tal como ocurre en la iglesia de Santiago y en el convento de los Loios (Arraiolos) (fig. 4). Tratándose, las dos últimas, de obras de mayor envergadura, se plantea nuevamente, a manera de corroboración, la cuestión de las colaboraciones subyacentes en obras de su autoría.

En lo concerniente al tratamiento de determinados elementos, merecen una referencia especial - como no podría ser de otro modo- los rostros, duros, como de campesinos, según defendía Robert Smith, pero que son, pese a sus divergencias y a la falta de consistencia que denotan- una de las improntas más singulares de la obra de Barco. Los rostros masculinos, toscos y escuálidos, presentan un tratamiento cuidado. El intento de individualizarlos es evidente. Los femeninos, sin embargo, varían entre dos tipos que se repiten sistemáticamente: de perfil o frontales, pero siempre con un trazo sintético, mientras que los muy cuidados se van renovando apenas con ligeras variantes a lo largo de toda su obra. También las manos, muy perfiladas, huesudas y a veces con las puntas de los dedos afiladas, algo cónicas, surgen de forma constante en toda su producción.

El paisaje, o los árboles y la vegetación, presentan motivos semejantes (hojas acaracoladas, hojas rayadas, troncos rellenos con pinceladas rápidas), pero tan variables que no constituyen una sólida base de apoyo. En los fondos arquitectónicos, en cambio, se constatan las dificultades del autor a la hora de definir la perspectiva y el planteamiento de las composiciones en el espacio. 
Esta caracterización abreviada nos ha permitido percibir, entre otras cosas, que la obra de Barco no conoció una evolución significativa ${ }^{54}$, sino que osciló entre tendencias en las que predominaba el sentido expresivo, pictórico y manchado, a veces incluso con borrones de pintura. Con todo, habría que señalar los cambios que se registran entre el Barco de aquella primera obra de Barcarena y el Barco de una de sus últimas obras, localizada en Portalegre (fig. 2). El descubrimiento del grabado que sirvió de fuente de inspiración para los dos paneles, ambos con la representación del Bautismo de Cristo, permite realizar una comparación sobre bases bastante más sólidas. Así, mientras que las figuras -particularmente la de Cristo- surgen achatadas y desequilibradas en 1691, en los paneles de la capilla particular, pintados en 1700, se respira una agilidad y una seguridad propias sólo de quien posee un dominio avezado del dibujo, la pintura y la composición. Con todo, no olvidemos que, ese mismo año, Barco había pintado los paños que revestirían el convento de los Loios, donde predomina un espíritu más tosco y descuidado. En conclusión, Barco sabe muy bien, desde el primer momento, qué y cómo pintar, pero opta por adaptar su técnica y ejecución según el encargo que se trae entre manos. ¿Será que en el origen de estas opciones tan dispares lo que encontramos es una valoración previa del público y del cliente o será que esa disparidad se debe a condicionantes derivadas de la copia de los grabados utilizados y de su fiel reproducción?

En efecto, el grabado constituye un elemento fundamental en el proceso de construcción de los revestimientos de azulejos figurativos de este periodo. Aunque la composición de los cuadros cerámicos estuviesen inspirados en ellos, los artistas utilizan estas inagotables fuentes de inspiración de una forma libre, adaptando las escalas, retirando o añadiendo figuras, haciendo más simples o complejos los escenarios, invirtiendo las imágenes, además de otras muchas opciones.

Todos los grabados utilizados por Barco e identificados hasta el momento -algunos de ellos inéditos- muestran trazos paralelos que conducen inevitablemente a relacionarlos con la forma de pintar analizada anteriormente. Por otro lado, los grabados de caza de Antonio Tempesta - usados en el palacio de la Independencia y en la casa del Terreiro do Trigo- son más esquemáticos y contienen menos información que los restantes. Las planchas más complejas, como las de Pietro Testa utilizadas por Barco en los dos conjuntos de Évora, fueron, no obstante, muy alteradas en los azulejos de la iglesia de Santiago, tanto a través del enriquecimiento de los escenarios arquitectónicos -como ocurre con el Regreso del hijo pródigo- como de la supresión de motivos probablemente considerados inapropiados, como ocurre con la escena que retrata a $E l$ hijo pródigo en casa de las cortesanas. En Beja, el Descanso durante la fuga a Egipto, inspirado en una estampa de Pier Francesco Mola, denuncia problemas de composición mal resueltos, aunque la riqueza del modelo dotó de mejor calidad al azulejo, tal como sucedería en Barcarena y Portalegre, copias fieles del grabado de Cornelis Cort que respetan todas las zonas de luz y sombra. En muchos de estos modelos también se pueden apreciar árboles con follaje acaracolado junto a troncos dinámicos que, con toda probabilidad, dejaron huella en el pintor.

La inconstancia de la obra de Gabriel del Barco plantea otras interrogantes a propósito de sus colaboradores asiduos y ocasionales, por desgracia aún no identificados. Trabajos como los que realizó para la iglesia de Santiago de Évora o para el convento de los Loios, en años consecutivos, implican, naturalmente, la ayuda o colaboración de otros pintores. Barco puede que haya aprendido con artistas de la generación anterior, con los que habría colaborado, pero, sin duda alguna, también formó a muchos otros contemporáneos suyos. Estas influencias mutuas ayudan a explicar la relativa ingenuidad que se aprecia en la pintura de azulejos de finales del siglo XVII, por contraste con la obra erudita de los designados Mestres [maestros], cuya formación les permitió

${ }^{54}$ Como ya había referido Meco, José, “Azulejos de Gabriel del Barco...”, op. cit., 1979, p. 79. 
solucionar los problemas de perspectiva y de dibujo que caracterizan a sus antecesores. Con todo, conviene no olvidar que António de Oliveira Bernardes fue contemporáneo de Gabriel del Barco, y que ambos se conocían no sólo por la obra que realizaron en colaboración en la ciudad de Beja -Bernardes se encargó del techo y de la pintura al óleo-, sino también porque ambos eran miembros de la Hermandad de San Lucas, en la cual Bernardes entró un año después del español. Además, Bernardes ya había pintado azulejos en la década de 1690. De ello dan fe los paneles de la capilla de Nuestra Señora Do Monte (Quinta da Ramada, parroquia de Frielas), actualmente en la Casa de Santa María (Cascáis).

De estas quince obras cuya atribución a Barco es segura, nueve fueron realizadas para Lisboa, una para los alrededores y las restantes cinco para la región del Alentejo, o sea, el círculo de clientes del pintor, a diferencia de lo que sucedería más tarde con los pintores integrados en el denominado Ciclo dos Mestres, era mayoritariamente lisboeta, llegando como muy lejos hasta el Alentejo.

Por otro lado, Barco fue el artista de esta época que dejó un mayor número de paneles firmados. No se acaba de entender, sin embargo, qué habría llevado al pintor a estampar su nombre en unas obras y en otras no. ¿Una cuestión de orgullo? ¿Una marca de su superioridad o de la importancia del encargo?

\section{Una futura reapreciación de la obra de Gabriel del Barco}

La definición del corpus artístico de Gabriel del Barco se debe, sobre todo, a la labor de José Meco, quien, desde 1979, ha ido reuniendo en torno a este pintor un amplio conjunto de piezas cerámicas. También es cierto que Santos Simões ha establecido algunas atribuciones ${ }^{55}$, como ya habían hecho, aunque en menor número, algunos de los restantes autores mencionados, entre los que se cuentan Reynaldo dos Santos y Robert Smith.

Para apreciar mejor el acervo pictórico relacionado con Gabriel del Barco, hemos optado por crear un listado de las obras que en algún momento han sido relacionadas con el pintor español, independientemente de quién hiciese la atribución y de nuestra consideración sobre su grado de fiabilidad. El resultado ha sido impresionante, pues, además de los quince conjuntos documentados, se han asociado a la figura de Barco cerca de setenta obras más. Curioso es también analizar los números y la evolución de los mismos en el contexto de los estudios de cada investigador, o de los investigadores en su conjunto. Por ejemplo, Reynaldo dos Santos tan sólo añade cuatro más a las obras atribuidas con seguridad a Barco, mientras que Santos Simões eleva ese número a casi veinte. Por su parte, José Meco, en 1979, aumentó el número de núcleos cerámicos relacionados con Gabriel del Barco hasta veintisiete, aunque en un artículo suyo de ese mismo año - continuado en 1981- consiguió asociar a este pintor cuarenta y dos obras, entre las que se incluían, además de los paneles de autoría indiscutible, otros muchos, ya fueran obras en colaboración o trabajos relacionados o influidos de una u otra forma por Barco. Esta perspectiva inicial de José Meco fue, no obstante, conociendo ajustes a lo largo de los años y, en 1986 (1993), el número de obras había descendido ya a diecinueve, fijándose finalmente en dieciocho en el año 1989, época a la que remonta la última biografía exhaustiva de Barco, escrita precisamente por este investigador.

55 Cf. SimÕEs, João Miguel dos Santos, "Alguns azulejos de Évora”, en A Cidade de Évora, n. ${ }^{0}$ 5-10, vol. 2, Évora, 1945, publicado por LoPES, Vítor Sousa (coord.), Estudos de Azulejaria, 2001, Lisboa, Imprensa Nacional - Casa da Moeda, pp. 17-52; SIMÕES, João Miguel dos Santos, op. cit., 1979. 
Ante el panorama observado, y teniendo en consideración algunos estudios monográficos que han ido apareciendo, hemos creído urgente proceder a una reapreciación de este vasto conjunto de obras, procurando definir cuáles pueden o no ser efectivamente relacionadas con Gabriel del Barco, estableciendo conexiones entre ellas y sacando partido de la utilización de los medios tecnológicos disponibles actualmente.

De todas formas, dejemos esta materia para otro artículo y ciñámonos por ahora a destacar una vez más la importancia de Gabriel del Barco, pintor español al que se le debe reconocer un puesto puntero en el panorama de la historia de la azulejería portuguesa, en la que se destacó por su pintura expresiva e inmediata, una pintura superior a la producida por muchos de sus contemporáneos y que se acerca a la de los maestros del ciclo siguiente. Y así, haciéndole justicia, contribuir a dignificar una de las artes que más directamente se identifica con la singularidad del patrimonio artístico portugués.

Fecha de recepción: 19-XI-2010

Fecha de aceptación: 9-II-2011 